\title{
NEW PROPER MOTIONS FOR HIPPARCOS STARS
}

\author{
H. Jahreiß \\ Astronomisches Rechen-Institut W-6900 Heidelberg GERMANY \\ F. Crifo \\ Observatoire de Paris F-92195 Meudon FRANCE \\ and \\ $Y$. Réquième \\ Observatoire de Bordeaux F-33270 Floirac FRANCE
}

During the preparation of the Hipparcos Input Catalogue precise positions were measured very recently by the Bordeaux automatic meridian circle (BMC) for about 5500 stars in the northern hemisphere. On average eight observations were made for each star at a mean epoch 1987. The estimated mean error in $\alpha$ is 0.12 and in $\delta 0^{\prime \prime}$. 18. With 2555 stars almost half the BMC sample lies in the declination zone $-2^{\circ}<\delta<+32^{\circ}$. In this zone the $(x, y)$ measurements of all Astrographic Catalogue plates have been newly reduced (S. Röser, priv. comm.). So, at least two independent positions are available for practically all stars down to the limiting magnitude of the $\mathrm{AC}$ with epochs around 1910 and with a mean positional error of 0 ." 28 . A combination of these two sources should provide proper motions to an accuracy of $0 . .004 \mathrm{yr}^{-1}$.

Due to the lack of further information a computer programme was developed to cross-identify the two samples merely from the coordinates of the stars. A strategy was designed in order to reduce user intervention for double stars and other dubious identifications to a minimum.

$350 \mathrm{BMC}$ stars were probably below the magnitude limit of the $\mathrm{AC}$ plates and $126 \mathrm{BMC}$ stars were removed due to obvious duplicity. A total of $4462 \mathrm{AC}$ positions for $2079 \mathrm{BMC}$ stars could be identified. The resulting proper motions had a mean error 0." $003 \mathrm{yr}^{-1}$, slightly better than expected. Among these 1207 BMC stars had previously unknown proper motions. The values for 314 stars with only one $\mathrm{AC}$ position available should be regarded with care. A subsequent analysis of the resulting 48 new high-proper motion stars $\left(\mu \geq 0\right.$.' $\left.180 \mathrm{yr}^{-1}\right)$ showed 14 proper motions to be real, 25 proper motions require further investigation, and 9 proper motions to be spurious.

The present results demonstrate the great value of the Astrographic Catalogue as a reservoir of old epoch positions. Therefore, it is highly desirable that the remaining $\mathrm{AC}$ zones also become available soon in machine readable form. 